\title{
Assessment of right ventricular outflow tract fractional shortening as a measure of right ventricular function in chronic heart failure patients
}

\author{
*Nura Ibrahim Maiyadi 1,2, Ibraheem Faragallah ${ }^{1}$, Ahmad Abdelhameed Rozza ${ }^{1}$ \\ ${ }^{1}$ Department of Cardiology, Faculty of Medicine, Al-Azhar University, Egypt, ${ }^{2}$ Specialist Hospital \\ Sokoto, Sokoto State, Nigeria
}

*Corresponding author: Nura Ibrahim Maiyadi; Mobile: 01147793760; Email: nimdee030@ gmail.com

\begin{abstract}
:
Background: assessment of RV function is a major component of the management and prognostication of heart failure (HF) patients. Its complexity makes this task difficult and therefore not appropriately considered. Right ventricular outflow tract fraction (RVOT FS) can serve that purpose if done. The functional capacity of a HF patient must always be assessed and decision made on that basis.

Aim of the study: this study aimed to investigate the applicability of RVOT FS in assessment of RV function and also its relation to functional capacity.

Patients and methods: seventy-one (71) patients with heart failure with reduce ejection fraction (HFrEF) were studied prospectively and nineteen (19) control healthy individuals (normal ECG, normal left and right ventricular function and no cardiac risk factors). A 2D guided M-mode was taken in the parasternal short axis view to determine RVOT FS and a six-minute walk test (6MWT) was done to determine their functional capacity.

Results: there was a reduced RVOT FS reduced in the HFrEF group ( $p$ value $=0.005$ ), 6MWT D was reduced in the HFrEF group ( $p$ value $<0.001$ ), there was a positive correlation of RVOT FS with RVFAC $(\mathrm{r}=0.839, \mathrm{p}$ value $<0.001)$ TAPSE $(\mathrm{r}=0.830, \mathrm{p}$ value $<0.001), \mathrm{S}^{\prime}(\mathrm{r}=0.830, \mathrm{p}$ value $<0.001)$ 6MWT D $(r=0.953, p$ value $<0.001)$ and a negative correlation with $\operatorname{RIMP}(r=-0.867, p$ value $<0.001)$, RV FLS( $r=-0.878$, p value $<0.001)$, SPAP $(r=-0.633$, $\mathrm{p}$ value $<0.001)$. 6MWTD with $\operatorname{RVFAC}(\mathrm{r}=0.851$, $\mathrm{p}$ value $<0.001)$ TAPSE $(\mathrm{r}=0.825$, $\mathrm{p}$ value $<0.001)$, $\mathrm{S}^{\prime}(\mathrm{r}=0.837, \mathrm{p}$ value $<0.001)$, RVOT FS $(\mathrm{r}=0.953, \mathrm{p}$ value $<0.001)$ and a negative correlation with RIMP $(\mathrm{r}=-0.827$, $\mathrm{p}$ value $<0.001)$, RV FLS $(\mathrm{r}=-0.902$, $\mathrm{p}$ value $<0.001)$, SPAP $(\mathrm{r}=-0.621$, $\mathrm{p}$ value $<0.001)$. RVOT FS between HF subgroups significant ( $\mathrm{p}$ value $<0.001$ ).
\end{abstract}

Conclusion: RVOT FS is a simple and reliable parameter that can be used in assessment of RV function and has very positive correlation with functional capacity assessed by 6MWT.

Keywords: right ventricular outflow tract, chronic heart failure, right ventricular function

\section{Introduction:}

Chronic heart failure has recently been defined as Heart Failure (HF) diagnosed $\geq 3$ month duration $^{(\mathbf{1})}$. Recently heart failure has been classified into heart failure with reduce ejection fraction (HFrEF), with mid-range (HFmEF) and preserved ejection fraction $(\mathrm{HFpEP})^{(2)}$. It has long been established that clinical presentation and prognosis of heart failure patients is highly dependent on the RV function $^{(3-5)}$ The complexity of RV geometry has for long hindered the adequate assessment of RV function ${ }^{(6-9)}$. Right ventricular outflow tract fractional shortening is a new parameter that can be used to ease this distress ${ }^{(\mathbf{1 0 , 1 1})}$. Functional capacity is a major determinant and a prognostic factor in the life of a HF patient and this ought to be determined (12-14).

Aim of the work:
The aim of this study was to investigate the applicability of RV outflow tract fractional shortening (RVOT-FS) in the evaluation of RV function in patients with chronic HFrEF. Secondly to investigate the correlation between RVOT-FS and exercise capacity in patients with chronic HFrEF.

\section{Patients and methods:}

\section{Study design:}

The study was performed in Al Hussain University Teaching Hospital of Al Azhar University, Cairo-Egypt for 1year period from November 2017 to October 2018. This study was conducted prospectively on ninety individuals categorized into two groups. These groups were control group (19 individuals) healthy individuals (Normal ECG, normal left and right ventricular function ${ }^{(\mathbf{1 5})}$ and no cardiac 
risk factors) and HFrEF group (71 patients). Inclusion criteria was age > 18year, chronic heart failure diagnosed $\geq 3$ month, aetiology caused by coronary artery disease, or hypertensive heart disease, or idiopathic cardiomyopathy. Heart failure was defined as having HFrEF if left ventricular ejection fraction (LVEF) was $<40 \%{ }^{(2)}$. Exclusion criteria was patients with acute heart failure or heart failure < 3 months, all patients with significant arrhythmia, previous history of right heart failure or diagnosis of: group $1 \mathrm{PH}$ (e.g., pulmonary arterial hypertension), group $3 \mathrm{PH}$ ( $\mathrm{PH}$ associated with lung respiratory diseases and/or hypoxia), group $4 \mathrm{PH}$ (PH due to chronic thrombotic and/or embolic disease) and group 5 $\mathrm{PH}$ (PH associated with a miscellaneous of rare diseases) any orthopedic function or cognitive function impairment, poor echocardiographic window, age less than 18 year, more than moderate MR, and limiting neurological disease.

\section{Clinical history and examination}

Informed consent was taken from all patients for the study participation; careful history was taken from all patients for assessment of heart failure with emphasis on exercise tolerance of the patient, previous diagnostic tests done, mainly ECGs, echocardiography, nuclear scans, cardiac CT, CMR and coronary angiography. Focused clinical examination was done and a resting 12 lead ECG.

The HF patients were further classified into subgroup according to their exercise tolerance to New York heart association (NYHA) I-IV ${ }^{\mathbf{( 1 6 )}}$.

\section{Echocardiography:}

Standard transthoracic two-dimensional and Doppler echocardiographic examination was carried out with Philips iE33 X Matrix" ultrasound machine using "S5-1" matrix array transducer (Philips Medical Systems, Andover, USA) equipped with STE technology, using a multi frequency $(1-5 \mathrm{MHz})$. ECG-gated examination was done to optimal image acquisition and later analysis.

Left ventricular assessment: left ventricular end diastolic dimension (LVEDD) was determined by M-mode and left ventricular ejection fraction (LVEF) was determined by Simpson's biplane of disc method according to the American Society of Echocardiography(ASE) ${ }^{(15)}$.
Right ventricular assessment: RV focused view was obtained from the apical four chamber view and the following measurements were obtained according the $\mathrm{ASE}^{(7,15)}$. I) TAPSE: Was measured by M-mode with the cursor optimally aligned along the direction of the tricuspid lateral annulus and the systolic excursion distance measured with $<17 \mathrm{~mm}$ considered abnormal. II) RVFAC: this measurement was obtained by manually tracing the RV endocardial boarder in the systole and diastole of same frame, a value $<35 \%$ was considered abnormal. III) Right ventricular index myocardial performance (RIMP) and DTI-Derived Tricuspid Lateral Annular Systolic Velocity (S) were measured by aligning color tissue Doppler cursor to the lateral TV annulus and recording the maximum systolic velocity(S) and from one heartbeat RIMP $=($ IVRT + IVCT $) /$ ET $=($ TCO-ET $) / E T$. IV). Right ventricular free wall longitudinal strain (RV FLS) manual tracing of the endocardial border of the RV over single frame the endocardial borders where automatically be tracked throughout the cardiac cycles. V). Systolic pulmonary artery pressure (SPAP) was estimated by measuring peak systolic tricuspid gradient and adding estimated RAP by measuring the IVC. ${ }^{(15)}$

Right ventricular outflow tract fractional shortening (RVOT FS), a 2D zoomed guided M-mode is obtained in parasternal short axis view in the distal RVOT, end diastole and end systole using endocardial leading-edge to obtain the as RVOT FS $\%=($ RVOT ED RVOT ES)x100/(RVOT ED) ${ }^{(\mathbf{1 0})}$.

Six-minute walk test distance (6MWT D), patients were asked to walk for 6 minute at their own pace in a corridor of about $10 \mathrm{~m}$ to and fro, the distance covered was then calculated after wards..$^{(17)}$

\section{Statistical analysis:}

Obtained data were recorded in Microsoft excel work sheet and analyzed using SPSS 20.0, categorical data was presented as frequencies and percentage, while continuous data were presented in mean $\pm \mathrm{SD}$. Chi square was used to analyze categorical data, independent T-test was used to compare continuous data in the two groups, analysis of variance(ANOVA) with Turkey significance was used to compare the NYHA groups and a Pearson or Spearman 
correlation was calculated to show the relation between variables.

\section{Results:}

Demographic and risk factors data showed no statistically significant difference between the two groups, Age 49.58 \pm 8.83 in control vs $50.87 \pm 6.94$ in $\mathrm{HF}$ with $\mathrm{P}$ value $=0.498$, Gender 10 out of 19 were male in control and 39 out of 71 were male with a $\mathrm{p}$ value $=0.858$, Smoking, Hypertension and Diabetes mellitus had $\mathrm{P}$ value $=0.391, \mathrm{P}$ value $=0.102$ and $\mathrm{P}$ value $=0.083$ respectively.

\begin{tabular}{|l|l|l|l|}
\hline \multicolumn{1}{|l}{ Table 1: demographic and risk factors between the two groups } & \\
\hline Group & Group 1(control) & Group 2(HF) & P value \\
\hline Age & $49.58 \pm 8.83$ & $50.87 \pm 6.94$ & 0.498 \\
\hline Gender & 10 M vs 9 F & 39 M vs 32 F & 0.858 \\
\hline Smoking & Yes 5 vs No 14 & Yes 25 vs No 46 & 0.391 \\
\hline hypertension & No (19) & Yes 9 vs No 62 & 0.102 \\
\hline Diabetes mellitus & No (19) & Yes 10 vs No 61 & 0.083 \\
\hline & & & \\
\hline
\end{tabular}

There was a statistically significant difference in the echocardiographic findings, included RVOT FS and 6MWT D between the two groups with a significant $\mathrm{p}$ value as shown in table 2 .

\begin{tabular}{|c|c|c|c|}
\hline PaTEInts & Control (19) & HF (71) & $P$ value \\
\hline LVEF \% & $64.32 \pm 5.67$ & $25.44 \pm 6.86$ & $<0.001$ \\
\hline LVEDD (mm) & $48.16 \pm 4.94$ & $69.96 \pm 5.98$ & $<0.001$ \\
\hline $\mathbf{S}^{\prime}(\mathbf{m m} / \mathbf{s})$ & $15.32 \pm 2.43$ & $11.25 \pm 4.32$ & $<0.001$ \\
\hline RIMP & $0.41 \pm 0.045$ & $0.52 \pm 0.10$ & $<0.001$ \\
\hline RV FLS & $-25.16 \pm 2.79$ & $-16.07 \pm 7.79$ & $<0.001$ \\
\hline eSPAP (mmHg) & $10.63 \pm 6.64$ & $48.80 \pm 14.58$ & $<0.001$ \\
\hline 6MWT (m) & $582.79 \pm 61.61$ & $296.59 \pm 176.37$ & $<0.001$ \\
\hline RVOT FS (\%) & $48.39 \pm 7.11$ & $37.54 \pm 16.11$ & 0.005 \\
\hline TAPSE (mm) & $24.95 \pm 3.86$ & $17.32 \pm 6.10$ & $<0.001$ \\
\hline RVFAC \% & $43.74 \pm 4.55$ & $33.85 \pm 11.2$ & $<0.001$ \\
\hline
\end{tabular}

There was a statistically significant correlation between the RVOT FS and all other RV systolic parameters and also 6MWT D as shown in table 3.

\begin{tabular}{|l|c|l|}
\hline \multicolumn{2}{|l|}{ Table 3- Pearson correlation between the RVOT FS and other RV systolic parameters and functional capacity } \\
\hline ROVT FS & Pearson Correlation $(\mathbf{r})$ & P Value \\
\hline TAPSE & 0.830 & $<0.001$ \\
\hline RVFAC & 0.839 & $<0.001$ \\
\hline S' & 0.830 & $<0.001$ \\
\hline RIMP (TIE Index) & -0.867 & $<0.001$ \\
\hline RV FLS & -0.878 & $<0.001$ \\
\hline SPAP & -0.633 & $<0.001$ \\
\hline 6MWT D & 0.953 & $<0.001$ \\
\hline
\end{tabular}

There was a significant correlation between functional capacity of the patients with their RV function including the RVOT FS and there was no correlation with the LVEF and LVEDD as shown in table 4.

Table 4: Pearson correlation between the functional capacity, RV systolic parameters and left ventricular parameters

\begin{tabular}{|l|l|l|}
\hline 6MWT D & Pearson Correlation $(\mathbf{r})$ & P Value \\
\hline LVEF & 0.086 & 0.477 \\
\hline LVEDD & -0.010 & 0.996 \\
\hline TAPSE & 0.825 & $<0.001$ \\
\hline RVFAC & 0.851 & $<0.001$ \\
\hline S & 0.837 & $<0.001$ \\
\hline RIMP $($ TIE Index) & -0.827 & $<0.001$ \\
\hline RV FLS & -0.902 & $<0.001$ \\
\hline SPAP & -0.621 & $<0.001$ \\
\hline RVOT FS & 0.953 & $<0.001$ \\
\hline
\end{tabular}


There was no statistically significant difference between NYHA class in respect of their LVEF and LVEDD, but there was a highly statistically significant difference between these classes regarding their RV systolic parameters including RVOT FS and 6MWT D as shown in table 5.

\begin{tabular}{|l|l|l|l|l|l|}
\hline \multicolumn{2}{l}{ Table 5: comparison between HF subgroups regarding echocardiographic findings } \\
\hline Heart failure & $\begin{array}{l}\text { NYHA I } \\
\text { N=27 }\end{array}$ & $\begin{array}{l}\text { NYHA II } \\
\text { N=12 }\end{array}$ & $\begin{array}{c}\text { NYHA III } \\
\text { N=14 }\end{array}$ & $\begin{array}{c}\text { NYHA IV } \\
\text { N=18 }\end{array}$ & P Value \\
\hline LVEF & $25.44 \pm 6.21$ & $25.17 \pm 5.89$ & $26.79 \pm 6.47$ & $24.56 \pm 8.81$ & 0.842 \\
\hline LVEDD & $70.11 \pm 6.0$ & $70.75 \pm 5.94$ & $68.71 \pm 4.70$ & $70.17 \pm 7.12$ & 0.842 \\
\hline TAPSE & $21.78 \pm 3.96$ & $20.67 \pm 5.40$ & $14.29 \pm 3.99$ & $10.78 \pm 2.39$ & $<0.001$ \\
\hline RVFAC & $42.44 \pm 5.96$ & $40.50 \pm 10.72$ & $27.79 \pm 6.35$ & $21.22 \pm 3.72$ & $<0.001$ \\
\hline S & $14.67 \pm 3.33$ & $13.50 \pm 2.844$ & $8.64 \pm 1.906$ & $6.67 \pm 1.414$ & $<0.001$ \\
\hline RIMP & $0.45 \pm 0.050$ & $0.48 \pm 0.07$ & $0.56 \pm 0.09$ & $0.63 \pm 0.04$ & $<0.001$ \\
\hline RV FLS & $-22.22 \pm 2.17$ & $-20.92 \pm 3.00$ & $-13.14 \pm 6.16$ & $-5.89 \pm 3.740$ & $<0.001$ \\
\hline SPAP & $41.56 \pm 8.74$ & $38.75 \pm 9.91$ & $52.07 \pm 15.03$ & $63.83 \pm 10.93$ & $<0.001$ \\
\hline ROVT FS & $52.96 \pm 3.80$ & $45.03 \pm 5.37$ & $28.26 \pm 8.69$ & $16.61 \pm 4.30$ & $<0.001$ \\
\hline
\end{tabular}

\section{Discussion:}

RV has been a neglected entity for quite long by the scientific and cardiology community ${ }^{(6,7)}$, this has recently changed due to emerging evidence of its role in major cardiopulmonary diseases $^{(\mathbf{1 8 )}}$ and more importantly a prognostic factor in HF mortality $\left.{ }^{(3,4)}\right)^{(19)}$. Functional capacity in HF patients and response to CRT has been closely related to RV function not $\mathrm{LVEF}^{(\mathbf{2 0 , 2 1})}$. Determination of RV function has been a nightmare to the cardiologist due to the $3 \mathrm{D}$ complex structure of $\mathrm{RV}^{(7)}$, making the need for CMR or Radionuclide studies as gold standards in assessment of RV function ${ }^{(\mathbf{8 , 9})}$. RVOT FS has been suggested to be a good marker of RV function and also prognostic

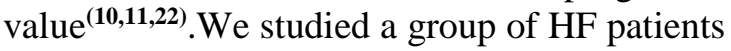
in comparison to control and found a significantly reduced RVOT FS along with other parameters in compared to the control group, which is similar to the findings of some author of Lindquist et al. ${ }^{(10)}$, Deveci et al. ${ }^{(23)}$ and Allam et al. ${ }^{(24)}$. We also showed a reduction in functional capacity between the groups similar to the reports of Lipkin et al. ${ }^{(25)}$, who also compared HF patients with the control. We found a very significant correlation between RVOT FS and all other RV parameters taken in this study even more correlate with RIMP, RV FLS and RVFAC which were less angle dependent. These findings are similar to those of Lindquist et al. ${ }^{(10)}$, Deveci et al. $^{(23)}$ and Allam et $a .^{(24)}$ except none of the mentioned studies took all the validated RV parameters and none compared to RVOT FS with RV FLS.
We also found a very significant correlation between RVOT FS and functional capacity of our study patients by using 6MWT which is so far the first study to make this observation to the best of Our knowledge. This is in conformity the known fact that RV function is a major determinant of HF patient functional capacity. There was also no any correlation between the RVOT FS and LVEF in our study, this similar to the findings of Allam et al. ${ }^{(24)}$ and contrary to Yamaguchi et $a l .{ }^{(11)}$ and Deveci et $a l .{ }^{(23)}$, Asmer et al. $^{(26)}$, this could be explained by different population and geographic group studied by the two studies mentioned.

We studied the functional capacity of the HF patients in relation their LV and RV parameters and found no any correlation between the functional capacity and LVEF and LVEDD, which is similar to the findings of Deveci $\boldsymbol{e t}$ $\boldsymbol{a l} .{ }^{(23)}$ though they used NYHA functional classification as their parameter for functional capacity. It is similar to results of Naibé et al. ${ }^{(27)}$ findings who conducted 6MWT in patients with HF. This is in disagreement to the findings of Wegrzynowska-Teodorczyk et $\boldsymbol{a l}^{(28)}$, who demonstrated a statistically significant relation, this could possibly be due to difference in race and geographic population of study. There was a very significant correlation between 6MWT $\mathrm{D}$ and all the studied RV parameters and this in total agreement with the findings of some authors as Di Salvo et al. ${ }^{(29)}$, Yamaguchi et $a l .{ }^{(11)}$, Deveci et $a l .{ }^{(23)}$, Ghio et $a l .{ }^{(3)}$, Meyer et $a l .{ }^{(30)}$, Ghio et al. $.^{(19},{ }^{31)}$, Guazzi et al. ${ }^{(19)}$, Kjaergaard et $a .^{(32)}$, WegrzynowskaTeodorczyk et al. ${ }^{(28)}$. 
And finally, we demonstrated a significant statistical relation between NYHA class and RV systolic parameters (TAPSE, RVFAC, eSPAP, RIMP (Tie index), S', RV FLS and RVOT FS). This is in agreement with findings of Bulent Deveci et al ${ }^{(23)}$, Yamaguchi et al..$^{(11)}$, Srinivasan et al. ${ }^{(33)}$, Meyer et al. ${ }^{(30)}$, Passantino et al. ${ }^{(34)}$. Though NYHA seemed not to have any relation with LVEF or LVEDD of our patients which is contrary to the findings of Wegrzynowska-Teodorczyk et al. $^{(28)}$.

\section{Limitations of this study:}

This study was limited by it being a single centre study, not use of CMR which is the gold standard in the assessment of RV function but this is due to constraint in the available resources. Cardiopulmonary exercise was not used as the gold standard in assessing functional capacity but 6MWT has received several validations for that purpose. The study is also limited by a relatively small sample size.

\section{Conclusion:}

RVOT FS is a simple non-invasive echocardiographic parameter that can be used to assess RV systolic function with a high degree of precision and can be used solely or in addition to other parameters. It may also give some prognostic information on the patient.

Conflict of interest: none

Funding and sponsorship: this study did not receive any financial support in any form.

\section{References:}

1. Atherton JJ, Sindone A, De Pasquale CG et al. (2018): National Heart Foundation of Australia and Cardiac Society of Australia and New Zealand: Australian clinical guidelines for the management of heart failure 2018. Med J Aust., 27: 1123-1208

2. Ponikowski P, Voors A (2016): 2016 Esc guidelines for the diagnosis and treatment of acute and chronic heart failure: The Task Force for the diagnosis and treatment of acute and chronic heart failure of the European society of cardiology (ESC) Eur. Heart J. , 37:2129-200.

3. Ghio S, Gavazzi A, Campana $\mathrm{C}$ et al. (2001): Independent and additive prognostic value of right ventricular systolic function and pulmonary artery pressure in patients with chronic heart failure. J Am Coll Cardiol .,37(1):183-8

4. Ghio S, Guazzi M, Scardovi AB et al. (2017): Different correlates but similar prognostic implications for right ventricular dysfunction in heart failure patients with reduced or preserved ejection fraction. Eur J Heart Fail, 19(7):873-879.

5. H L, Lam CSP, Gong L et al. (2017): Right ventricular dysfunction in leftsided heart failure with preserved versus reduced ejection fraction. Eur $\mathrm{J}$ Heart Fail, 19(12):1664-1671.

6. Greyson CR (2011): Evaluation of right ventricular function. Curr Cardiol Rep., 13(3):194-202.

7. Rudski LG, Lai WW, Afilalo J et al. (2010): Guidelines for the Echocardiographic Assessment of the Right Heart in Adults: A Report from the American Society of Echocardiography. Endorsed by the European Association of Echocardiography, a registered branch of the European Society of Cardiology, and . J Am Soc Echocardiogr, 23(7):685-713.

8. Wu VC-C, Takeuchi M (2018): Echocardiographic assessment of right ventricular systolic function. Cardiovasc Diagn Ther., 8(1):70-79.

9. Buechel ERV, Mertens LL (2012): Imaging the right heart: The use of integrated multimodality imaging. Eur Heart J., 33(8):949-60.

10. Lindquist $\mathbf{P}$, Henein $\mathbf{M}$, Kazzam $\mathbf{E}$ (2003): Right ventricular outflow-tract fractional shortening: An applicable measure of right ventricular systolic function. Eur J Echocardiogr, 4(1):2935.

11. Yamaguchi $\mathbf{M}$, Tsuruda $\mathbf{T}$, Watanabe Y et al. (2013): Reduced fractional shortening of right ventricular outflow tract is associated with adverse outcomes in patients with left ventricular dysfunction. Cardiovasc Ultrasound, 11(1):1-8.

12. Davies EJ, Moxham T, Rees K et al. (2010): Exercise based rehabilitation for heart failure. In: Cochrane Database of Systematic Reviews, 12(7):706-15.

13. Juenger $\mathbf{J}$ (2002): Health related quality of life in patients with congestive heart failure: comparison with other chronic 
diseases and relation to functional variables. Heart, 87(3):235-41.

14. Abraham WT, Fisher WG, Smith AL et al. (2002): Cardiac Resynchronization in Chronic Heart Failure. N Engl J Med., 346(24):184553.

15. Lang RM, Badano LP, Mor-Avi V et al. (2015): Recommendations for cardiac chamber quantification by echocardiography in adults: An update from the American society of echocardiography and the European association of cardiovascular imaging. Eur Heart J Cardiovasc Imaging, 16(3):233-271.

16. Levin R, Dolgin M, Fox C, Gorlin R (1994): The Criteria Committee of the New York Heart Association. https://books.google.com.eg/books?isb $\mathrm{n}=0190225505$

17. ATS (2002): Guidelines for the sixminute walk test. Am J Respir Crit Care Med., 166(1):111-117.

18. Marcu CB, Beek AM, Van Rossum AC (2006): Cardiovascular Magnetic Resonance Imaging for the Assessment of Right Heart Involvement in Cardiac and Pulmonary Disease. Hear Lung Circ., 15(6):362-70.

19. Guazzi M, Bandera F, Pelissero G et al. (2013): Tricuspid annular plane systolic excursion and pulmonary arterial systolic pressure relationship in heart failure: an index of right ventricular contractile function and prognosis. AJP Hear Circ Physiol., 305(9):H1373-81.

20. Damy T, Kallvikbacka-Bennett A, Goode K et al. (2012): Prevalence of, associations with, and prognostic value of tricuspid annular plane systolic excursion (TAPSE) among out-patients referred for the evaluation of heart failure. J Card Fail, 18(3):216-25.

21. Alpendurada F, Guha K, Sharma R et al. (2011): Right ventricular dysfunction is a predictor of nonresponse and clinical outcome following cardiac resynchronization therapy. J Cardiovasc Magn Reson., 31;13:68.

22. Lindqvist $\mathbf{P}$, Calcutteea $\mathbf{A}$, Henein $\mathbf{M}$ (2008): Echocardiography in the assessment of right heart function. Eur J Echocardiogr, 9(2):225-234.
23. Deveci B, Baser K, Gul Met al. (2016): Right ventricular outflow tract function in chronic heart failure. Indian Heart J., 68:S10-S14.

24. Allam L, Onsy A, Ghalib H (2017): Right ventricular outflow tract systolic excursion and fractional shortening: Can these echocardiographic parameters be used for the assessment of right ventricular function? J Cardiovasc Echogr., 27(2):52.

25. Lipkin DP, Scriven AJ, Crake T, Poole-Wilson PA (1986): Six minute walking test for assessing exercise capacity in chronic heart failure. $\mathrm{Br}$ Med J ., 292(6521):653-5.

26. Asmer I, Adawi S, Ganaeem M, Shehadeh J, Shiran A (2012): Right ventricular outflow tract systolic excursion: A novel echocardiographic parameter of right ventricular function. Eur Heart J Cardiovasc Imaging, 13(10):871-877.

27.

https://www.scitechnol.com/...review /functional-capacity-assessment-inpatients-with-c

28. Wegrzynowska-Teodorczyk K, Rudzinska E, Lazorczyk M et al. (2013): Distance covered during a sixminute walk test predicts long-term cardiovascular mortality and hospitalisation rates in men with systolic heart failure: An observational study. J Physiother, 59(3):177-187.

29. Di Salvo TG, Mathier M, Semigran MJ, Dec GW (1995): Preserved right ventricular ejection fraction predicts exercise capacity and survival in advanced heart failure. J Am Coll Cardiol., 25(5):1143-53.

30. Meyer P, Filippatos GS, Ahmed MI, et al. (2010): Effects of right ventricular ejection fraction on outcomes in chronic systolic heart failure. Circulation, 121(2):252-258.

31. Damy T, Ghio S, Rigby AS et al. (2013): Interplay between right ventricular function and cardiac resynchronization therapy: An analysis of the care-HF trial (Cardiac resynchronization-heart Failure). J Am Coll Cardiol., 61(21):2153-60.

32. Kjaergaard J, Petersen CL, Kjaer A, Schaadt BK, Oh JK, Hassager C 
Assessment of right ventricular outflow tract fractional shortening as a measure of right...

(2006): Evaluation of right ventricular volume and function by $2 \mathrm{D}$ and $3 \mathrm{D}$ echocardiography compared to MRI. Eur J Echocardiogr., 7(6):430-8.

33. Srinivasan A, Kim J, Khalique O et al. (2017): Echocardiographic linear fractional shortening for quantification of right ventricular systolic function-A cardiac magnetic resonance validation study. Echocardiography, 34(3):348-
358.

34. Passantino A, Lagioia R, Mastropasqua F, Scrutinio D (2006): Short-Term Change in Distance Walked in 6 Min Is an Indicator of Outcome in Patients With Chronic Heart Failure in Clinical Practice. J Am Coll Cardiol, 48(1):99-105. 